for

\title{
Development of Nanostructured Lipid Carriers for the Delivery of Idebenone in Autosomal Recessive Spastic Ataxia of Charlevoix-Saguenay
}

Chiara Martinelli ${ }^{1}$, Matteo Battaglini ${ }^{1,2}$, Carlotta Pucci $^{1}$, Sara Gioi ${ }^{3}$, Chiara Caracci $^{3}$, Gaia Macaluso ${ }^{4}$, Stefano Doccini ${ }^{5}$, Filippo M. Santorelli ${ }^{5}$, Gianni Ciofani ${ }^{1}$

${ }^{1}$ Istituto Italiano di Tecnologia, Smart Bio-Interfaces, Viale Rinaldo Piaggio 34, 56025 Pontedera, Italy

${ }^{2}$ Scuola Superiore Sant'Anna, The Biorobotics Institute, Viale Rinaldo Piaggio 34, 56025 Pontedera, Italy

${ }^{3}$ Politecnico di Torino, Department of Mechanical and Aerospace Engineering, Corso Duca degli Abruzzi 24, 10129 Torino, Italy

${ }^{4}$ Università di Pisa, Dipartimento di Biologia, Via Luca Ghini 13, 56126 Pisa, Italy

${ }^{5}$ IRCCS Stella Maris, Molecular Medicine for Neurodegenerative and Neuromuscular Diseases Unit, Via dei Giacinti 3, 56128 Calambrone (Pisa), Italy

\section{Corresponding Authors}

*chiara.martinelli@,iit.it; gianni.ciofani@,iit.it 

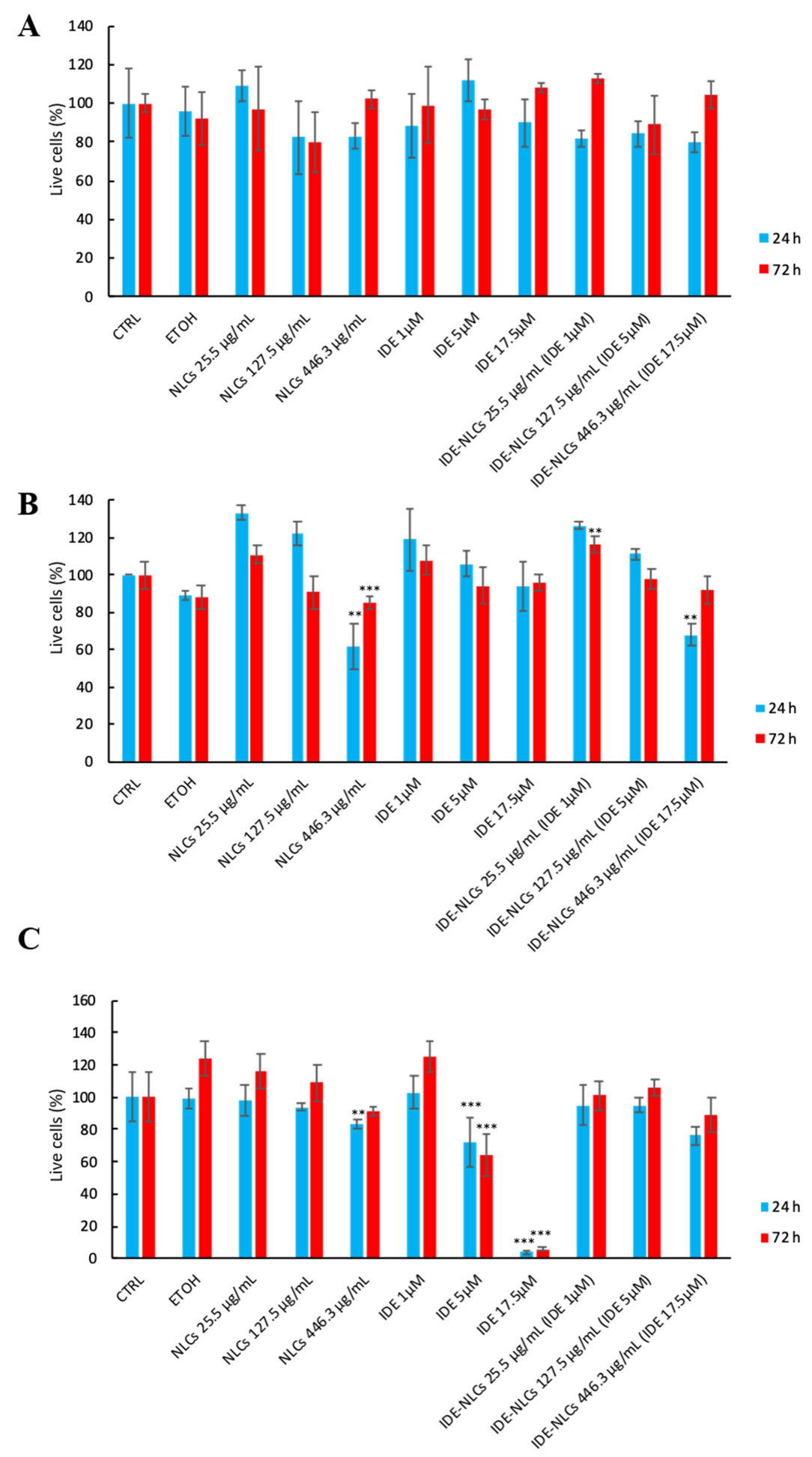

Figure S1. A) PicoGreen assay performed on C8-D1A astrocytes incubated with free IDE, NLCs, and IDE-NLCs. B) PicoGreen assay performed on bEnd.3 endothelial cells incubated with free IDE, NLCs, and IDE-NLCs. C) PicoGreen assay performed on differentiated SH-SY5Y neuron-like cells incubated with free IDE, NLCs, and IDE-NLCs. Analyses were normalized on non-treated cells (CTRL); cells were also treated with ethanol (ETOH, used for dissolving free idebenone). ${ }^{*} p$ $<0.05, * * p<0.01, * * * p<0.001$. 


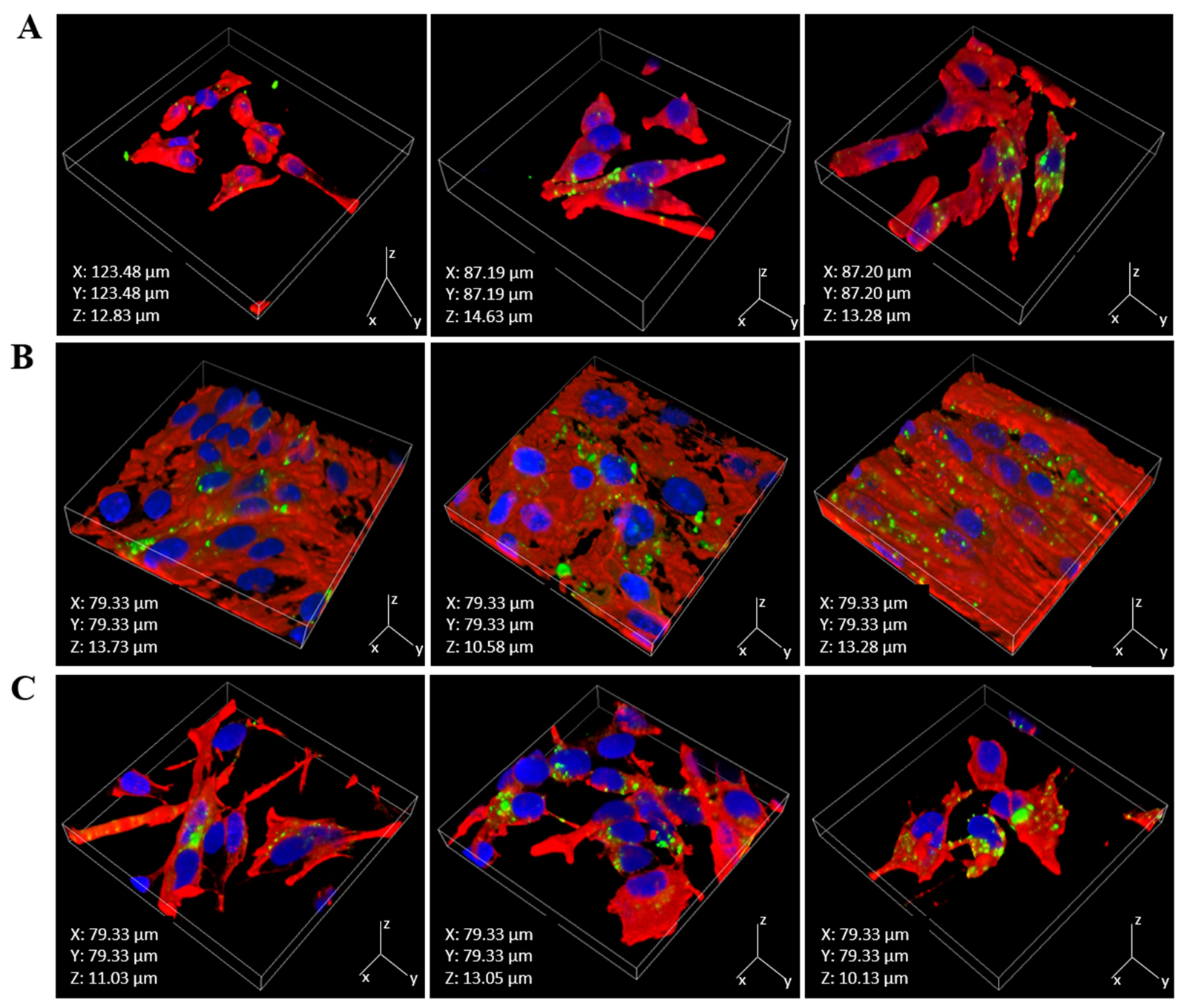

Figure S2. A) 3D merged CLSM images of C8-D1A astrocytes treated for $24 \mathrm{~h}$ (left), $48 \mathrm{~h}$ (middle), and $72 \mathrm{~h}$ (right) with Vybrant DiO-labeled IDE-NLCs (green). Nuclei (blue) and f-actin (red) were also stained. B) 3D merged CLSM images of bEnd.3 endothelial cells treated for $24 \mathrm{~h}$ (left), $48 \mathrm{~h}$ (middle), and $72 \mathrm{~h}$ (right) with Vybrant DiO-labeled IDE-NLCs (green). Nuclei (blue) and f-actin (red) were also stained. C) 3D merged CLSM images of differentiated SH-SY5Y neuron-like cells treated for $24 \mathrm{~h}$ (left), $48 \mathrm{~h}$ (middle), and $72 \mathrm{~h}$ (right) with Vybrant DiO-labeled IDE-NLCs (green). Nuclei (blue) and f-actin (red) were also stained. 
A
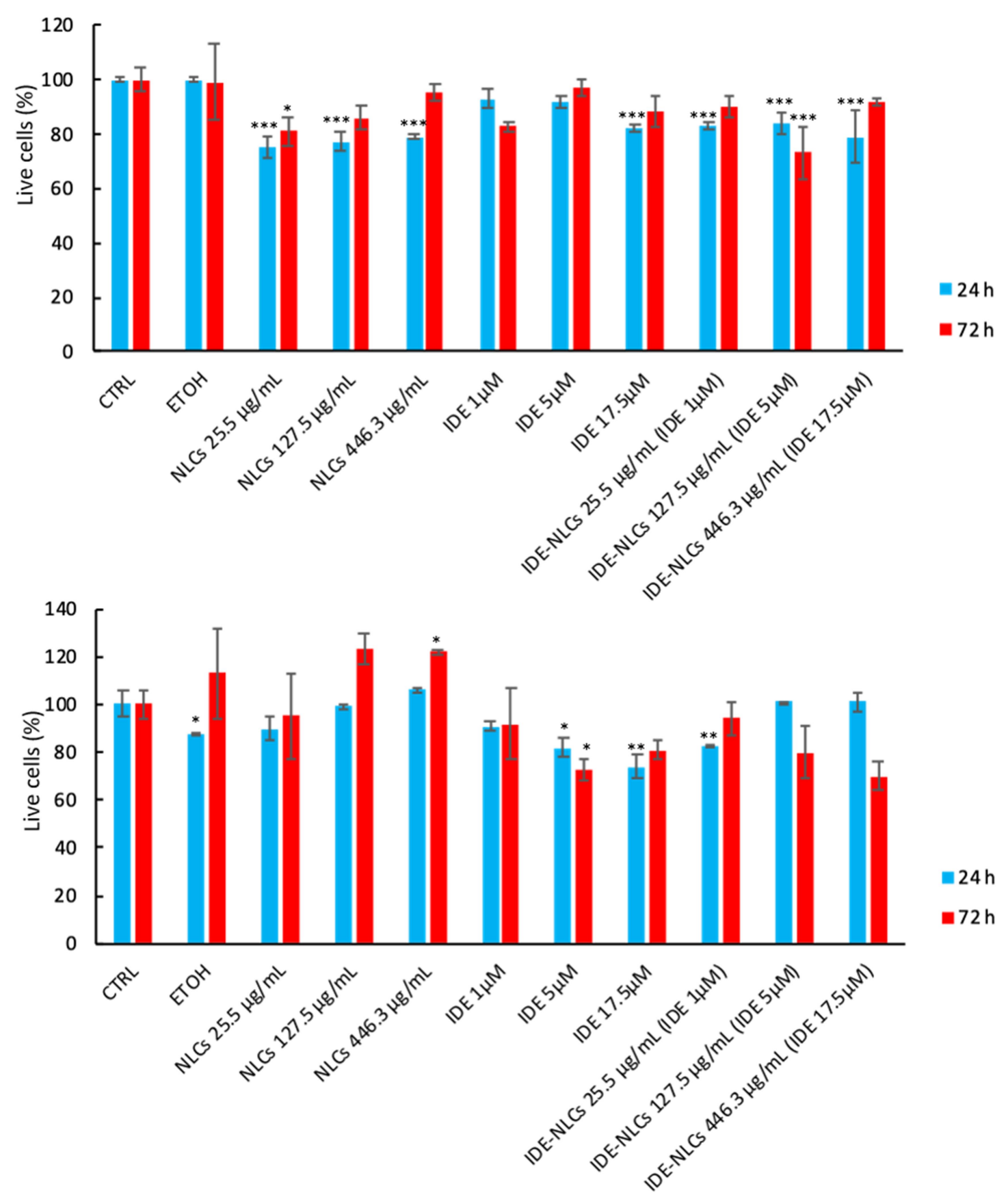

Figure S3. A) PicoGreen assay performed on human healthy primary fibroblasts incubated with free IDE, NLCs, and IDE-NLCs. B) PicoGreen assay performed on human ARSACS patient's primary fibroblasts incubated with free IDE, NLCs, and IDE-NLCs. Analyses were normalized on non-treated cells (CTRL); cells were also treated with ethanol (ETOH, used for dissolving free idebenone). $* p<0.05, * * p<0.01, * * * p<0.001$. 

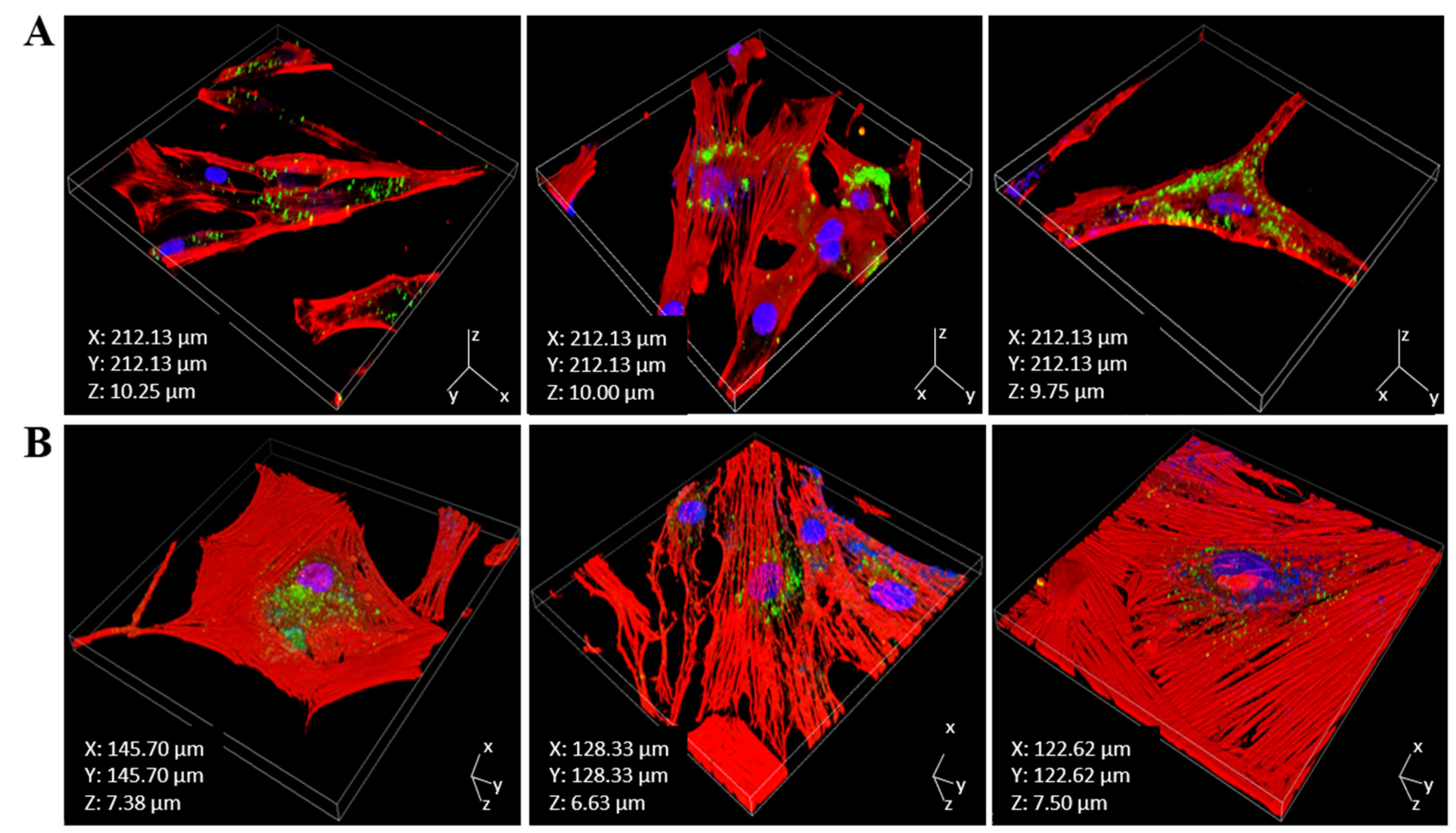

Figure S4. A) 3D merged CLSM images of human healthy primary fibroblasts treated for $24 \mathrm{~h}$ (left), $48 \mathrm{~h}$ (middle), and $72 \mathrm{~h}$ (right) with Vybrant DiO-labeled IDE-NLCs (green). Nuclei (blue) and f-actin (red) were also stained. B) 3D merged CLSM images of human ARSACS patient's primary fibroblasts treated for $24 \mathrm{~h}$ (left), $48 \mathrm{~h}$ (middle), and $72 \mathrm{~h}$ (right) with Vybrant DiO-labeled IDE-NLCs (green). Nuclei (blue) and f-actin (red) were also stained. 
A
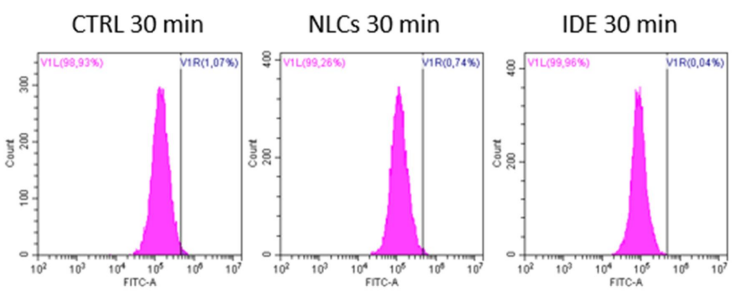

IDE-NLCs $30 \mathrm{~min}$

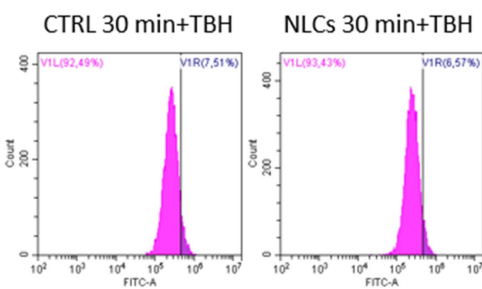

IDE $30 \mathrm{~min}+\mathrm{TBH}$
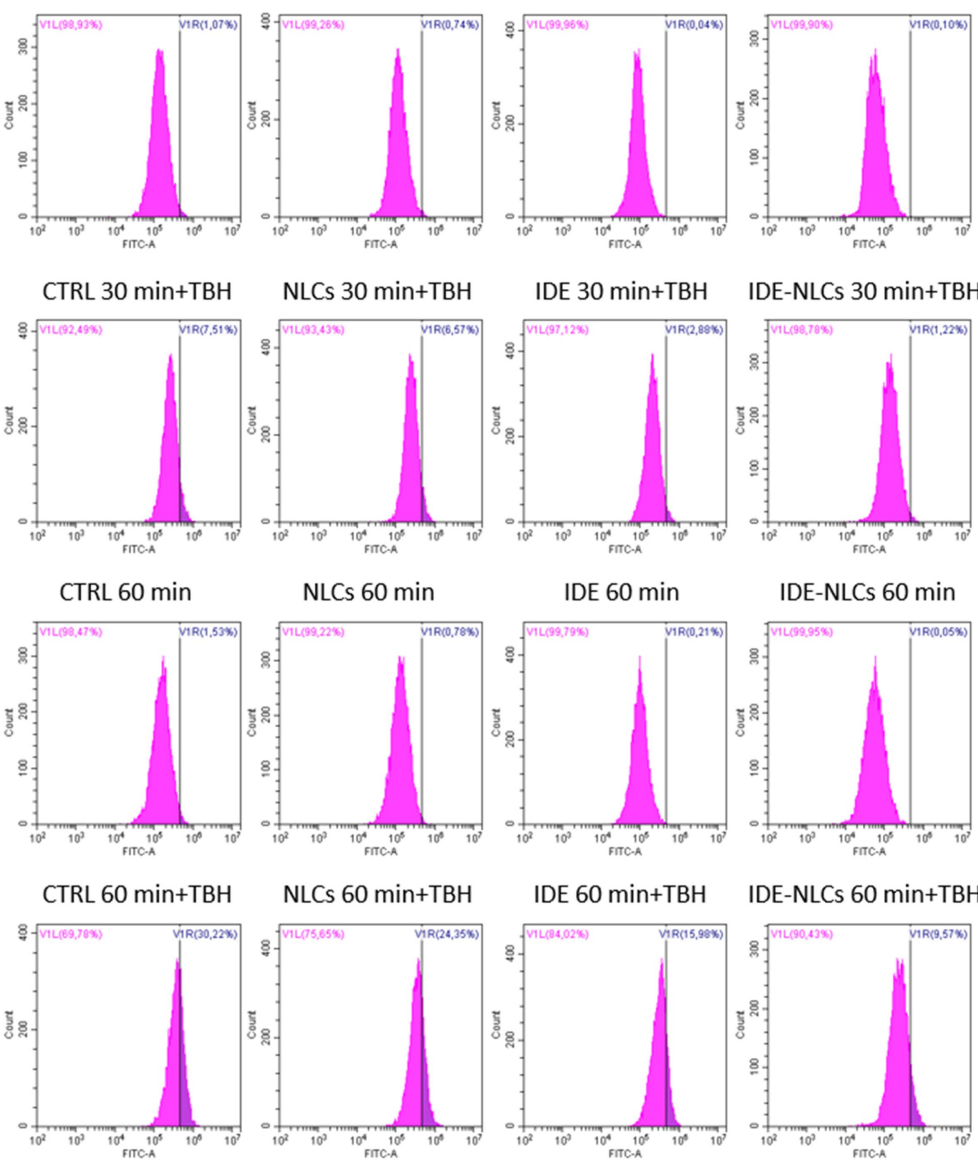

IDE $60 \mathrm{~min}+\mathrm{TBH}$

IDE-NLCS $60 \mathrm{~min}$
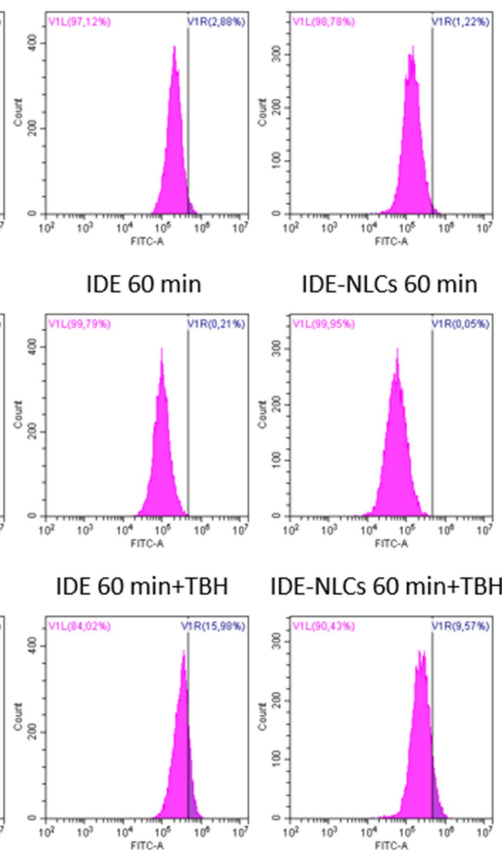

IDE-NLCs $60 \mathrm{~min}+\mathrm{TBH}$

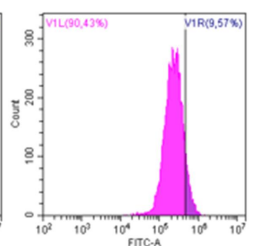

B
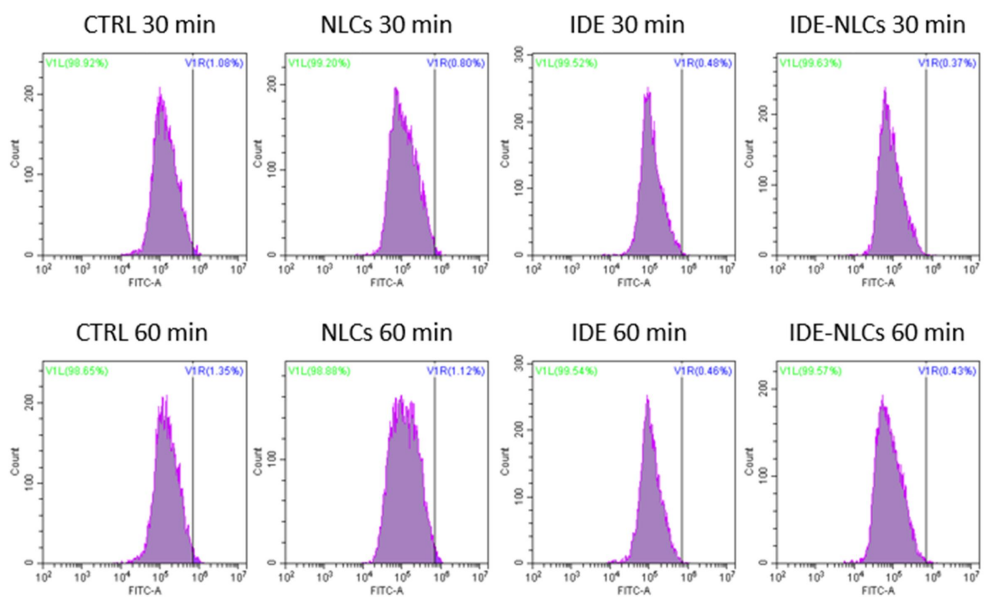

IDE-NLCs $60 \mathrm{~min}$

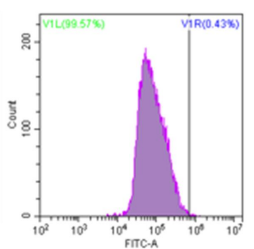

Figure S5. ROS detection through CellRox. A) Representative flow cytometry plots obtained for human healthy primary fibroblasts treated with free IDE, NLCs, and IDE-NLCs for $72 \mathrm{~h}$, acquired $30 \mathrm{~min}$ and $60 \mathrm{~min}$ after induction of oxidative stress by TBH. B) Representative flow cytometry plots obtained in ARSACS patient's primary fibroblasts treated with free IDE, NLCs, and IDENLCs for $72 \mathrm{~h}$, acquired $30 \mathrm{~min}$ and $60 \mathrm{~min}$ after cell detachment. 